\title{
Inter- and Intra-Rater Reliability of the Australian Spasticity Assessment Scale in Adults with Acquired Brain Injury
}

\author{
Anya Calame1, Barbara J. Singer ${ }^{2 *}$ \\ ${ }^{1}$ Physiotherapy Department, Sir Charles Gairdner Hospital, Nedlands, Perth, Australia \\ ${ }^{2}$ The Centre for Musculoskeletal Studies, School of Surgery, The University of Western Australia, Perth, \\ Australia \\ Email: barbara.singer@uwa.edu.au
}

Received 28 May 2015; accepted 24 July 2015; published 27 July 2015

Copyright (C) 2015 by authors and Scientific Research Publishing Inc.

This work is licensed under the Creative Commons Attribution International License (CC BY). http://creativecommons.org/licenses/by/4.0/

(c) $\underset{\mathrm{EY}}{\mathrm{B}}$ Open Access

\begin{abstract}
Objective: This study investigated the inter- and intra-rater reliability of the Australian Spasticity Assessment Scale (ASAS) in adults with unilateral hypertonia following acquired brain injury. The ASAS has been shown to be superior to other clinical tools for the assessment of spasticity in children with cerebral palsy but reliability has not been previously examined in adults. Method: Four muscle groups were rated on one occasion by four assessors using the ASAS in sixteen adults with unilateral hypertonia following acquired brain injury. Twelve participants returned one week later for reassessment by the same assessors. Results: Overall inter-rater reliability of the ASAS using a quadratic weighted Kappa was moderate $\left(K_{q w} 0.58\right)$ with ranges from moderate to good $\left(K_{q w} 0.42-0.70\right)$. Agreement between raters was greatest for soleus muscle and least for wrist flexors. Overall intra-rater reliability of each of the four raters was moderate to good $\left(K_{q w} 0.48\right.$ 0.79). Agreement within raters was greatest for soleus muscle and least for biceps muscle. Conclusions: The ASAS may represent an appropriate alternative to the clinical scales currently used to assess spasticity; however inter and intra-rater reliability data from this investigation are lower than those which have previously been reported by experienced users of the ASAS in children with cerebral palsy. Further investigation with a larger sample size is warranted before any firm conclusions may be drawn about the reliability and validity of this tool to assess spasticity in adults with acquired brain injury.
\end{abstract}

\section{Keywords}

Adult, Measurement, Spasticity, Australian Spasticity Assessment Scale, Inter-Rater Reliability, Intra-Rater Reliability

\footnotetext{
${ }^{*}$ Corresponding author.

How to cite this paper: Calame, A. and Singer, B.J. (2015) Inter- and Intra-Rater Reliability of the Australian Spasticity Assessment Scale in Adults with Acquired Brain Injury. Open Journal of Therapy and Rehabilitation, 3, 77-86. 


\section{Introduction}

Hypertonia, traditionally defined as greater than expected resistance to lengthening of a relaxed muscle [1], is observed in a variety of adult onset neurological conditions. Hypertonia results from rheologic changes within the muscle, as well as muscle overactivity due to disordered neural control [2]. Three subtypes of neurologically mediated hypertonia have been described in childhood disorders: spasticity, dystonia, and rigidity [3]. In adults, a widely accepted definition of spasticity is: “...a motor disorder characterised by a velocity-dependent increase in tonic stretch reflexes (muscle tone) with exaggerated tendon jerks, resulting from hyper excitability of the stretch reflex, as one component of upper motor neuron syndrome.” ([4] p. 182).

The reported prevalence of spasticity in adult conditions affecting the upper motor neurone is variable, with overall prevalence post stroke ranging from $4 \%$ to $42.6 \%$, whereas disabling symptoms are reported to be present in $2 \%-13 \%$ of stroke survivors with this impairment [5]. One reason for this wide variation in prevalence is likely to be the range of measurement tools utilised to evaluate spasticity [6] [7]. For instance, Wissel et al. [5] reported that up to $43 \%$ of patients, assessed using the Modified Ashworth Scale (MAS), experienced spasticity post ischemic stroke, whereas only $24 \%$ of patients within 12 months post-stroke were identified as having spasticity in the stroke affected arm when assessed using laboratory-based measures of hyper-reflexia (electromyography and torque) [8].

In some of the literature exploring various treatments for spasticity, there is a lack of coherence between the definition of spasticity and the measures used [6]. In the case of the most widely accepted definition of spasticity from Lance [4], valid and reliable measurements to characterize increased reflex responses are needed in order that treatment can be targeted appropriately to this aspect of hypertonia. Despite a general consensus for the need of a more quantitative scale, there is no recognized "gold standard" clinical measure of spasticity [9]. Failure to establish precisely the contribution of spasticity to hypertonia may mean that patients are unnecessarily exposed to the risks and side effects associated with oral anti-spastic medications [10], or may not be offered appropriate treatment such as botulinum toxin injection [11] [12]. Additionally, imprecise measurement tools may lead to an under-estimation of the contribution of spasticity to disability and impact on the evaluation of the effectiveness of management options for this impairment [6] [9] [13].

Numerous scales to identify and quantify spasticity have been developed. The most frequently used clinical methods for estimation of spasticity are the Ashworth Scale (AS) [14], the Modified Ashworth Scale (MAS) [15], the Tardieu Scale (TS) [16], first published in English by Gracies et al. [17] and the Modified Tardieu Scale (MTS) [18]. The AS and the MAS are unable to differentiate the contribution of velocity-dependent responses (spasticity) to the increased muscle resistance due to the single velocity application of these tests. In addition, the AS and the MAS have been shown to be poorly related to electromyographic data and to be better related to biomechanical measures of hypertonia [19] [20]. There are limited data to support the reliability of either of these scales, with somewhat better results for the upper limb compared with the lower limb [20]-[25]. Consequently, several researchers have concluded that the reliability of the AS and the MAS is insufficient for them to be a useful measure of spasticity [20] [23] [24]. The TS and the MTS have been suggested to be more appropriate clinical measures of spasticity as both of these scales involve assessment of resistance to passive movement at both slow and fast speeds [17] [18] [26]. Good to excellent reliability has been demonstrated for the TS and the MTS in the assessment of spasticity in children and adults (reviewed by [27]). One potential area of inconsistency when using these scales relates to the use of a goniometer to determine the "spasticity angle" (X). In one study of the reliability of the TS, agreement between examiners was defined as "an absolute difference equal to or less than $10^{\circ}$ ” ([26], p. 424) which points to the potential for considerable inter-rater variation in identifying the "catch point" [28]. In addition to recording the spasticity angle, the TS allows the quality of the response to rapid passive lengthening to be graded, using a hierarchical set of categories from "clear catch at precise angle, interrupting passive movement, followed by release" to "unfatigable clonus (>10 s when maintaining pressure) occurring at a precise angle” ([26], p. 427).

A recent evaluation of the MTS concluded that this scale is reliable for assessing spasticity in most lower limb muscles of adults with chronic neurologic injuries; however "repeated MTS measurements of spasticity are best based on $R 1$ measurements rather than spasticity angle or qualitative ratings of spasticity” ([27], p. 2494). A further criticism of the TS and MTS is that they do not account for other sources of resistance to rapid passive movement such as those resulting from adaptations in the morphology of the musculotendinous unit, for instance changes in soft tissue compliance and adaptive shortening [2]. 
This study explores the clinical utility, as well as inter- and intra-rater reliability of a novel scale- the Australian Spasticity Assessment Scale (ASAS) which was developed to overcome perceived shortcomings of existing scales in assessing spasticity in the clinical setting in children [29] [30]. The ASAS is based on the framework of the MAS and the MTS, and conforms to the definitions of spasticity proposed by Lance [4] and Sanger et al. [3] as the muscle response to a series of rapid stretches through full available range of motion is scored. This tool has a prescribed test procedure, is quick and easy to perform, and has an unambiguous scoring system (see Table 1).

Validity and inter-rater reliability of the ASAS have been shown to be superior to other clinical tools available for the assessment of spasticity in children with cerebral palsy (CP) [29] [30], but reliability has not been previously examined in adults. In a study by Love et al. [30], elbow flexors, wrist flexors, and forearm pronator muscle groups were assessed in the upper limb, while adductors, hamstrings, gastrocnemius and tibialis posterior muscles were assessed in the lower limb. Of the 314 muscle groups analysed in 23 children there was complete agreement in ASAS score between three raters for 238 muscles (75.8\% of tests) while only two raters agreed on the ASAS score for further 70 muscles (22.3\% of tests). However, disagreements tended to represent only one ASAS category. Lower limb muscles demonstrated very good reliability overall ( $\kappa_{q w}$ range: $0.81-0.84$ ); whereas upper limb reliability varied from good to very good for each individual muscle ( $\kappa_{q w}$ range: 0.75 0.92). An intra-class correlation (ICC) was also calculated to allow comparison with the literature on other clinical scales to assess spasticity in children and also showed very good reliability for the ASAS (ICC 0.88 : $95 \%$ CI 0.86 to 0.90 ) [30].

The present study is the first, to our knowledge, to explore inter and intra-rater reliability of the ASAS in adults with spasticity following acquired brain injury (ABI).

\section{Methods}

\subsection{Study Participants}

\subsubsection{Subjects}

A sample of convenience including people aged between 18 and 65 years, who were living in the community or in supported accommodation and met the following criteria: able to give informed consent, stable unilateral spasticity following an ABI greater than 6 months since onset, and not currently undergoing intensive therapy, were invited to participate. Participants were excluded from the study if they had any musculoskeletal impairment that would limit available passive range of movement (e.g. severe pain, fixed contracture) or had had Botulinum toxin injection, or commenced on any new anti-spasticity medications, in the three months prior to data collection.

\subsubsection{Raters}

Four physiotherapists experienced in acute neurology, but who did not regularly use clinical scales to assess spasticity, participated in this study. Gonimetric data were collected by physiotherapy students. Several training workshops, conducted by the developers of this scale, were held prior to data collection. The training days focused on: 1) positioning accuracy for each of the four muscle groups identified for the study; 2) accurate use of goniometry; 3) procedural consistency for the method of application of the ASAS; and 4) resolving any disagreement about allocation of an ASAS score. In addition, regular practice sessions were scheduled during the weeks prior to data collection to familiarise all assessors with the scale and the test procedure. The ASAS was

Table 1. Australian spasticity assessment scale-scoring criteria [30].

0

1

2

3

4
No catch on rapid passive movement (RPM) [i.e. no spasticity].

Catch occurs on RPM followed by release. There is no resistance to RPM throughout rest of range.

Catch occurs in second half of available range (after halfway point) during PRM and is followed by resistance throughout remaining range.

Catch occurs in first half of available range (up to and including halfway point) during RPM and is followed by resistance throughout the remaining range.

When attempting RPM, the body part appears fixed but moves on slow passive movement.

Note: contracture is recorded separately. 
administered as per procedures demonstrated in the DVD provided by the authors of the scale.

\subsection{Ethics}

This study was approved by the Human Research Ethics Committee at North Metropolitan Health Service, WA Health, Western Australia. All participants signed written informed consent forms prior to participation.

\subsection{Data Collection}

The data collected comprised two joint angles (maximal passive muscle length-R2), and the angle at which a catch was consistently detected (R1) which was tested as per the procedure for the TS outlined by Gracies et al. [26]. Subsequent to the administration of the TS, an ASAS score was allocated for four muscles on the affected side: hamstrings (assessed via the popliteal angle); soleus (assessed via ankle dorsiflexion with the knee in $90^{\circ}$ flexion); biceps (assessed via elbow extension with the forearm in neutral rotation); and wrist flexors as a group (assessed via wrist extension with the forearm in neutral rotation and the elbow flexed to $90^{\circ}$ ).

Where possible, participants were measured on two occasions. The time interval between repeated measurements was seven days, to ensure enough time between measurements so that the rating physiotherapist was unable to recall previous scores, but not so long as to allow a potential change in spasticity due to an alteration in the participant's status for other reasons. Participants were tested on the same day of the week and at the same time of day to minimize any confounding influences on the results of change of activity levels or diurnal variation in spasticity.

\section{Test Procedures}

Participants were positioned in supine and the test area was configured to minimise environmental stimulation. Careful attention was paid to standardizing the overall posture (including neck position) and position of adjacent joints. Bony landmarks for goniometry were marked on all participants by an independent person using standardised criteria. The rater performed the test manoeuvre and then maintained the limb position while the goniometric measure was performed. All goniometric measurements were rounded up to the nearest degree. ASAS raters were blinded to the goniometric data. Maximal passive muscle length (R2) was tested first. No discussion of results was permitted between raters or student recorders. Participants received a five minute break between assessments in which they were allowed to sit up if desired. Rater order was consistent between and within study participants.

1) Allocating an $R 2$ score: The muscle/muscle group was moved passively through its full excursion from its shortest anatomical position. The speed of the stretch was very slow and the stretch was sustained to ensure that maximal range of motion was achieved. Maximal muscle length was considered to be represented by the joint angle achieved following a sustained stretch and was recorded in degrees by the student recorder, but was not communicated to the examiner administering the stretch.

2) Allocating an $R 1$ score: The muscle/muscle group to be tested was passively returned to its shortest anatomical position. Three rapid passive movements were performed through the available range of motion as fast as the examiner could move the limb, without exerting force. The angle at which the rapid passive movement was interrupted by a stretch induced muscle contraction (catch or clonus, if present) was measured in degrees as per the documented procedure for the TS [26]. The examiner administering the stretch was blinded to the angle recorded.

3) Allocating an ASAS score: A fourth rapid passive movement, from the same starting position, was performed in the same direction and at the same velocity, to confirm the previously identified point of catch in the arc of motion, but using enough force to move the muscle/muscle group past the catch angle to the available end range. The examiner determined the location of the catch or clonus (if present) and the presence or absence of passive resistance after the point of catch, and allocated an ASAS grade accordingly (Table 1) which was recorded on the assessment sheet by the student assisting with data collection.

\subsection{Data Analysis}

Inter and intra-rater reliability were calculated using the weighted kappa statistic (quadratic weighting). A weighted kappa statistic was chosen due to the ordinal nature of the scale. The quadratic weighted kappa is con- 
sidered to approximate the intra-class correlation coefficient for scales with 5 or more categories. Analysis was performed overall, and for each muscle group. The results for the weighted kappa were interpreted as follows: very good $0.81-1.00$, good $0.61-0.80$, moderate $0.41-0.60$, fair $0.21-0.41$, and poor $<0.21$ [31]. Any outcomes with a confidence interval crossing 0 , or a p value $<0.05$ were not rated. All statistical analyses were performed using Stata10 Version 10.1 (Stat Corp, Texas).

\section{Results}

Data for sixteen individuals are included. Twelve subjects who were able to attend for a second test were measured twice, one week apart. All of the participants had hemiplegia secondary to stroke or trauma (11 had occlusive stroke and 5 had suffered a haemorrhagic event). An equal number of subjects were included with left and right hemiplegia. The average age of participants was 48.3 (range 27 - 62 years). The average duration of hemiplegia was 61.7 months (range 7 - 420 months). Fourteen participants were living in the community, the remaining two subjects were in a residential care facility.

\subsection{Inter Rater Reliability-4 Raters}

The distribution of ASAS scores across the four muscle groups tested and the four raters are presented in Table 2. All ASAS scores were represented. Data are presented in Table 3 for inter-rater reliability for the four raters, overall and for each muscle group on one test occasion across sixteen subjects. Overall inter-rater reliability of

Table 2. Distribution of ASAS scores ( $\mathrm{n}=16$ subjects).

\begin{tabular}{|c|c|c|c|c|c|}
\hline $\mathrm{N}=16$ subjects & ASAS 0 & ASAS 1 & ASAS 2 & ASAS 3 & ASAS 4 \\
\hline Biceps Rater 1 & 1 & 1 & 9 & 5 & 0 \\
\hline Rater2 & 2 & 0 & 7 & 7 & 0 \\
\hline Rater3 & 2 & 0 & 4 & 10 & 0 \\
\hline Rater4 & 1 & 0 & 12 & 3 & 0 \\
\hline Wrist flex R1 & 0 & 1 & 8 & 7 & 0 \\
\hline $\mathrm{R} 2$ & 3 & 0 & 11 & 2 & 0 \\
\hline R3 & 0 & 0 & 9 & 7 & 0 \\
\hline $\mathrm{R} 4$ & 0 & 3 & 5 & 8 & 0 \\
\hline Hamstrings R1 & 1 & 3 & 9 & 3 & 0 \\
\hline $\mathrm{R} 2$ & 3 & 0 & 12 & 1 & 0 \\
\hline $\mathrm{R} 3$ & 1 & 0 & 12 & 3 & 0 \\
\hline R4 & 6 & 0 & 6 & 4 & 0 \\
\hline Soleus R1 & 1 & 3 & 7 & 3 & 2 \\
\hline $\mathrm{R} 2$ & 5 & 1 & 8 & 1 & 1 \\
\hline R3 & 3 & 0 & 4 & 9 & 0 \\
\hline $\mathrm{R} 4$ & 4 & 1 & 3 & 6 & 2 \\
\hline
\end{tabular}

Table 3. ASAS inter rater reliability ( $\mathrm{n}=16$ subjects).

\begin{tabular}{|c|c|c|c|c|c|}
\hline & Time & Weighted Agreement (\%) & Expected Agreement (\%) & Kappa $\left(K_{q w}\right)$ & Agreement Rating \\
\hline Overall & $\mathrm{T} 1$ & 95.1 & 88.3 & $0.58(0.45,0.72)$ & Moderate \\
\hline Wrist Flex & $\mathrm{T} 1$ & 93.6 & 89.0 & $0.42(0.28,0.59)$ & Moderate \\
\hline Biceps & $\mathrm{T} 1$ & 93.8 & 85.6 & $0.56(0.10,1.26)$ & - \\
\hline Hamstrings & $\mathrm{T} 1$ & 88.9 & 80.6 & $0.43(0.05,0.90)$ & Moderate \\
\hline Soleus & $\mathrm{T} 1$ & 93.6 & 79.9 & $0.70(0.50,0.90)$ & Good \\
\hline
\end{tabular}


the ASAS was moderate $\left(K_{q w} 0.58\right)$ with ranges from moderate to good $\left(K_{q w} 0.42-0.70\right)$. Agreement between raters was greatest for soleus muscle and least for the wrist flexors.

\subsection{Intra Rater Reliability}

Data for the test re-test reliability for the four raters across twelve subjects, overall and by individual muscles are presented in Table 4. Overall intra-rater reliability for the four raters was moderate to good ( $K_{q w} 0.48$ - 0.79$)$. Agreement within raters was greatest for soleus muscle and least for biceps muscle.

\section{Discussion}

From this preliminary investigation of the use of the ASAS to assess spasticity in adults with unilateral hypertonia secondary to ABI, it would appear that this scale has moderate to good intra-rater agreement and inter-rater reliability. However there was a considerable range across the data, and test-retest data for some muscles had very wide confidence intervals (Table 2 and Table 3).

The ASAS was originally devised for the clinical assessment of spasticity in children with CP. The test procedure has many features in common with the TS and MTS. However, the ASAS allows both neural and nonneural contributors to rapid passive movement to be considered, by evaluating the "catch point" (spasticity) but also resistance throughout the remainder of the range (hypertonia). The categories of the ASAS also indicate where in the overall range the "catch" is felt. This could be considered to be a clinical correlate of the "reflex threshold" and reflects, to a certain extent, the "severity" of the velocity dependent response to rapid passive

\begin{tabular}{|c|c|c|c|c|c|}
\hline & Rater & $\begin{array}{l}\text { Weighted Agreement } \\
\text { (\%) }\end{array}$ & $\begin{array}{c}\text { Expected } \\
\text { Agreement (\%) }\end{array}$ & $\begin{array}{l}\text { Weighted Kappa } K_{q w} \\
(95 \% \text { CI) }\end{array}$ & Agreement Rating \\
\hline \multirow{4}{*}{ Overall } & 1 & 96.8 & 89.4 & $0.70(0.51,0.92)$ & Good \\
\hline & 2 & 97.6 & 89.4 & $0.77(0.61,0.95)$ & Good \\
\hline & 3 & 93.9 & 88.2 & $0.48(0.26,0.71)$ & Moderate \\
\hline & 4 & 96.9 & 85.5 & $0.79(0.67,0.92)$ & Good \\
\hline \multirow{4}{*}{ Wrist Flexors } & 1 & 89.6 & 83.3 & $0.38(-0.07,0.90)$ & - \\
\hline & 2 & 94.4 & 93.1 & $0.30(-0.60,0.84)$ & - \\
\hline & 3 & 83.3 & 54.2 & $0.64(0.11,1.21)$ & Good \\
\hline & 4 & 91.7 & 69.8 & $0.72(0.41,1.14)$ & Good \\
\hline \multirow{4}{*}{ Biceps } & 1 & 98.1 & 83.8 & $0.88(0.61,1.35)$ & - \\
\hline & 2 & 95.8 & 74.0 & $0.84(0.58,1,18)$ & - \\
\hline & 3 & 89.6 & 74.3 & $0.59(0.05,1.40)$ & - \\
\hline & 4 & 96.3 & 88.9 & $0.67(-0.14,2.04)$ & - \\
\hline \multirow{4}{*}{ Hamstrings } & 1 & 86.1 & 74.4 & $0.46(0.01,0.99)$ & - \\
\hline & 2 & 95.8 & 84.4 & $0.73(0.37,1,13)$ & - \\
\hline & 3 & 87.5 & 87.8 & $-0.03(-0.22,0.16)$ & - \\
\hline & 4 & 89.6 & 78.4 & $0.52(0.20,0.89)$ & Moderate \\
\hline \multirow{4}{*}{ Soleus } & 1 & 98.0 & 82.6 & $0.88(0.69,1.16)$ & Very good \\
\hline & 2 & 95.0 & 89.3 & $0.53(0.07,1.21)$ & Moderate \\
\hline & 3 & 90.9 & 82.6 & $0.48(0.08,0.95)$ & Moderate \\
\hline & 4 & 97.7 & 81.1 & $0.88(0.69,1.18)$ & Very good \\
\hline
\end{tabular}


movement. That is, if the muscle spindle is more sensitive to a stretch input, or relatively less inhibited, it will induce a 'reflex contraction' earlier in the lengthening procedure, indicating more severe spasticity. The scale is quick and easy to apply and the ASAS categories are tessellated, so that a muscle response to stretch should clearly fit into only one category.

Despite these potential advantages over other clinical scales, much lower reliability data were reported in this study than in previous investigations of the ASAS to assess spasticity in children with CP [29] [30]. There are a number of potential contributors to this finding. The 2013 report of the Australian Cerebral Palsy Register (birth years 1993-2006), suggests that spasticity was seen in almost 90\% of children with CP. In the study by Love et al. [30], all children evaluated had a primary diagnosis of spasticity; whereas the present study incorporated a relatively unselected population of adults with unilateral hypertonia related to ABI. Along with the presence of spasticity, co-existing soft tissue adaptation (muscle tightness and stiffness, but not fixed contracture), and spastic dystonia was seen in many subjects, as well as a degree of pain in the affected limbs in a few cases. The relatively limited pool of potential participants in the study, particularly for intra-rater evaluation, meant that subjects with some co-existing limb pain, joint and muscle stiffness, and other types of muscle overactivity, were included in the study sample, which may have confounded results. However this sample is representative of the type of co-impairments commonly seen in adults with ABI, for instance following stroke or traumatic brain injury.

A standardised procedure was used for data collection, which has been shown previously to improve the agreement between raters [26] [27]. Gracies et al. [26] also found that training regarding the application of the TS significantly improved the inter- and intra-rater reliability, even in experienced raters. This suggests that training may be beneficial for the application of any assessment scale, regardless of years of clinical experience. In the current study, the assessors received training and undertook considerable practice prior to data collection, however the assessors did not utilise clinical measures of spasticity on a daily basis, which may have distinguished them from the highly experienced paediatric physiotherapists who undertook data collection in the previous reliability studies by Love et al. [29] [30]. It is also important to note that, during the practice sessions, subjects were sourced from an acute neurology facility, where the early presentation of spasticity is rare, or practice was undertaken on normal individuals. Consequently it could be argued that the handling skills required to effectively apply the ASAS procedure in individuals with spasticity were not trained sufficiently in the raters used for this investigation.

One difficulty that is specific to the allocation of a score using the ASAS, relates to the estimation of whether the catch/clonus (if present) occurred in the second half or the first half of the range. It is the documented intention of the authors of the ASAS scale that this is an uninstrumented measure. In the present study, although formal measures of R1 and R2 were taken, as per the ASAS description, raters were blinded to the goniometric data and were therefore unable to use this information to estimate the midpoint in range. In cases where the catch/ clonus point occurred around mid-range, it was frequently difficult for raters to determine whether it fell before or at the midway point of the movement arc (ASAS score 3) or in the second half of the range (ASAS score 2). Compounding this difficulty was the fact that, depending on the degree of stiffness and the weight of the limb, raters frequently needed to hold the limb close to their body in order to stabilize it, while providing adequate force to move the limb through full available range at a sufficient velocity, thus reducing the rater's ability to determine accurately in what part of the arc of movement the catch had occurred. Positioning oneself to allow clear observation of the whole arc of the movement is likely to be much more difficult when assessing an adult compared with a child. In the previous reliability study [29] [30], children included had a mean age of approximately seven years, with a standard deviation of almost four years. It is likely that their limbs were, on average, smaller and easier to handle, making the ASAS easier to perform. However, this difficulty is not specific to the ASAS and would be encountered with many of the existing clinical scales currently employed to assess spasticity, such as the MTS and TS, where the rater has to arrest the movement at the point during a rapid passive stretch where a "catch" is felt, and maintain that position to allow a gonimetric measure to be made. This may be one reason why greater reliability has been reported for the TS in children [26] than adults with spasticity [27].

An additional difficulty in older adults is that pain from other sources, such as joint degeneration, may impact on the ability to relax the limb. This was one reason for restricting the test cohort to under 65 years of age. However several subjects in the present cohort did report some discomfort on testing, and in one case this resulted in the subject declining to attend for repeat assessment. Spasticity is a common impairment in older adults with conditions affecting the upper motor neurone, for instance stroke, and, as a result of the age limitations of 
the cohort tested, these data may not be representative of such populations.

The determination of the angle at which the catch point occurred may also have been altered by the administration of repeated passive stretches. The "catch" may have occurred earlier in the range during the passive stretches applied by the first rater, whereas thixotropic influences [32] may have lessened during subsequent assessments, leading successive raters to determine that the catch was in the second, rather than the first, half of the available range. This potential confounding influence was addressed by having a rest of five minutes between each assessment (and longer for a given muscle group as the order of muscle testing was kept constant). Post hoc analysis suggests that there was minimal influence of thixotropy on score allocation over the course of a single session, as a trend towards lower ASAS scores was not seen with successive assessments by each of the four raters.

The main limitation of this study was the small number of individuals included. The sample was one of convenience, drawn from a population of community dwelling adults with ABI, who were not currently receiving any therapy. The age restriction, imposed as outlined above in the expectation that it would minimise the presence of comorbidities, further limited the pool of available volunteers. In addition, the lack of comparison against a "gold standard" to explore the validity of the ASAS is also a significant limitation of the present study. A previous study investigating intra- and inter-rater reliability for the AS also explored the relationship between this clinical scale and quantitative measures of hypertonia (EMG and dynamometry) of the knee extensors and elbow flexors [20]. Particularly for the arm, there was little relationship between EMG data and AS scores, while relationships with biomechanical data were stronger, but still not highly correlated. Fleuren et al. [20] noted considerable variation in the velocity at which the passive stretch was applied both within and between raters. Variation in the velocity at which the stretch was applied is also likely to have occurred in the present study; however the influence of this potential source of variance is unable to be assessed, as no biomechanical data were collected from which to evaluate stretch velocity.

Finally, many previous studies have used two or three raters to determine inter-rater reliability and frequently have only evaluated agreement at only one or two muscle groups, with elbow flexors being the most commonly utilised [20] [24] [33]-[35]. The current study used four raters and evaluated four muscle groups on the affected side, including both large (hamstrings) and small (wrist flexors) muscle groups. While this allows a more comprehensive examination of the use of the ASAS, it exposes the data to many further sources of variation. The source of the considerable intra and inter-rater variation seen in ASAS scores is unable to be established from the present data. Further investigation, with a larger sample size, and utilising objective measures of both neural and non-neural contributors to passive muscle resistance is warranted before any firm conclusions may be drawn about the reliability and validity of the ASAS to measure spasticity in adults.

\section{Conclusion}

The ASAS was developed to address perceived shortcomings in existing clinical scales to assess spasticity in children. The scale's authors have reported good to very good reliability using this novel tool in a sample of children with CP with spasticity attending a tertiary centre. In this investigation to explore the applicability of the scale to adults, much lower inter- and intra-rater reliability was demonstrated. Further studies are needed in a larger sample to determine whether this scale is a useful addition to current clinical measures to assess spasticity in adults with upper motor neurone lesions.

\section{Acknowledgements}

Thanks are extended to the following people: study participants and carers, Noula Gibson for study development and manuscript review, Sarah Love for manuscript review, Natasha Bear for assistance with data analysis, Ian Cooper, Gillian Gregory, Cameron Mead, Amanda Ziatas, and physiotherapy students from Curtin University, Australia, and the University of Notre Dame Australia, for assistance with data collection and Isuru Patabendige for assistance with subject recruitment.

\section{References}

[1] Stolov, W.C. (1996) The Concept of Normal Muscle Tone, Hypotonia and Hypertonia. Archives of Physical Medicine and Rehabilitation, 47, 156-168.

[2] Singer, B., Dunne, J. and Allison, G. (2001) Reflex and Non-Reflex Elements of Hypertonia in Triceps Surae Muscles 
Following Acquired Brain Injury: Implications for Rehabilitation. Disability and Rehabilitation, 23, 749-757. http://dx.doi.org/10.1080/09638280110060466

[3] Sanger, T.D., Delgado, M.R., Gaebler-Spira, D., Hallett, M., Mink, J.W. and Task Force on Childhood Motor Disorders (2003) Classification and Definition of Disorders Causing Hypertonia in Childhood. Pediatrics, 111, e89-e97. http://dx.doi.org/10.1542/peds.111.1.e89

[4] Lance, J.W. (1980) Pathophysiology of Spasticity and Clinical Experience with Baclofen. In: Lance, J.W., Feldman, R.G., Young, R.R. and Koella, W.P., Eds., Spasticity: Disordered Motor Control, Year Book, Chicago, 185-204.

[5] Wissel, J., Verrier, M., Simpson, D.M., Charles, D., Guinto, P., Papapetropoulos, S. and Sunnerhagen, K.S. (2014) Post-Stroke Spasticity: Predictors of Early Development and Considerations for Therapeutic Intervention. PMR, 7, 6067.

[6] Malhotra, S., Pandyan, A.D., Day, C.R., Jones, P.W. and Hermens, H. (2009) Spasticity, an Impairment That Is Poorly Defined and Poorly Measured. Clinical Rehabilitation, 23, 651-658. http://dx.doi.org/10.1177/0269215508101747

[7] Wissel, J., Schelosky, L.D., Scott, J., Christe, W., Faiss, J.H. and Mueller, J. (2010) Early Development of Spasticity Following Stroke: A Prospective, Observational Trial. Journal of Neurology, 257, 1067-1072. http://dx.doi.org/10.1007/s00415-010-5463-1

[8] O’Dwyer, N.J., Ada, L. and Neilson, P.D. (1996) Spasticity and Muscle Contracture Following Stroke. Brain, 119, 1737-1749. http://dx.doi.org/10.1093/brain/119.5.1737

[9] Sunnerhagen, K.S., Olver, J. and Francisco, G.E. (2013) Assessing and Treating Functional Impairment in Poststroke Spasticity. Neurology, 80, S35-S44. http://dx.doi.org/10.1212/wnl.0b013e3182764aa2

[10] Lorentzen, J., Grey, M.J., Crone, C., Mazevet, D., Biering-Sørensen, F. and Nielsen, J.B. (2010) Distinguishing Active from Passive Components of Ankle Plantar Flexor Stiffness in Stroke, Spinal Cord Injury and Multiple Sclerosis. Clinical Neurophysiology, 121, 1939-1951. http://dx.doi.org/10.1016/j.clinph.2010.02.167

[11] Olver, J., Esquenazi, A., Fung, V.S., Singer, B.J., Ward, A.B. and Cerebral Palsy Institute (2010) Botulinum Toxin Assessment, Intervention and Aftercare for Lower Limb Disorders of Movement and Muscle Tone in Adults: International Consensus Statement. European Journal of Neurology, 17, 57-73. http://dx.doi.org/10.1111/j.1468-1331.2010.03128.x

[12] Sheean, G., Lannin, N.A., Turner-Stokes, L., Rawicki, B., Snow, B.J. and Cerebral Palsy Institute (2010) Botulinum Toxin Assessment, Intervention and After-Care for Upper Limb Hypertonicity in Adults: International Consensus Statement. European Journal of Neurology, 17, 74-93. http://dx.doi.org/10.1111/j.1468-1331.2010.03129.x

[13] Pandyan, A.D. (2002) Are We Underestimating the Clinical Efficacy of Botulinum Toxin (Type A)? Quantifying Changes in Spasticity, Strength and Upper Limb Function after Injections of Botox to the Elbow Flexors in a Unilateral Stroke Population. Clinical Rehabilitation, 16, 654-660. http://dx.doi.org/10.1191/0269215502cr536oa

[14] Ashworth, B. (1964) Preliminary Trial of Carisoprodol in Multiple Sclerosis. Practitioner, 192, 540-542.

[15] Bohannon, R. and Smith, M. (1987) Interrater Reliability of a Modified Ashworth Scale of Muscle Spasticity. Physical Therapy, 67, 206-207.

[16] Tardieu, G., Shentoub, S. and Delarue, R. (1954) Research on a Technic for Measurement of Spasticity. Revue Neurologique, 91, 143-144. (In French)

[17] Gracies, J.M., Marosszeky, J.E., Renton, R., Sandanam, J., Gandevia, S.C. and Burke, D. (2000) Short-Term Effects of Dynamic Lycra Splints on Upper Limb in Hemiplegic Patients. Archives of Physical Medicine and Rehabilitation, 81, 1547-1555. http://dx.doi.org/10.1053/apmr.2000.16346

[18] Boyd, R. and Graham, H.K. (1999) Objective Measurement of Clinical Findings in the Use of Botulinum Toxin Type A for the Management of Children with Cerebral Palsy. European Journal of Neurology, 6, S23-S35. http://dx.doi.org/10.1111/j.1468-1331.1999.tb00031.x

[19] Patrick, E. and Ada, L. (2006) The Tardieu Scale Differentiates Contracture from Spasticity Whereas the Ashworth Scale Is Confounded by It. Clinical Rehabilitation, 20, 173-182. http://dx.doi.org/10.1191/0269215506cr922oa

[20] Fleuren, J.F., Voerman, G.E., Erren-Wolters, C.V., Snoek, G.J., Rietman, J.S., Hermens, H.J. and Nene, A.V. (2010) Stop Using the Ashworth Scale for the Assessment of Spasticity. Journal of Neurology, Neurosurgery, and Psychiatry, 81, 46-52. http://dx.doi.org/10.1136/jnnp.2009.177071

[21] Allison, S.C., Abraham, L.D. and Petersen, C.L. (1996) Reliability of the Modified Ashworth Scale in the Assessment of Plantarflexor Muscle Spasticity in Patients with Traumatic Brain Injury. International Journal of Rehabilitation Research, 19, 67-78. http://dx.doi.org/10.1097/00004356-199603000-00007

[22] Haas, B., Bergström, E., Jamous, A. and Bennie, A. (1996) The Inter Rater Reliability of the Original and of the Modified Ashworth Scale for the Assessment of Spasticity in Patients with Spinal Cord Injury. Spinal Cord, 34, 560-564. http://dx.doi.org/10.1038/sc.1996.100 
[23] Gregson, J.M. and Sharma, A.K. (2000) Measuring Poststroke Spasticity. Reviews in Clinical Gerontology, 10, 69-74. http://dx.doi.org/10.1017/s0959259800001076

[24] Blackburn, M., Van Vliet, P. and Mockett, S.P. (2002) Reliability of Measurements Obtained with the Modified Ashworth Scale in the Lower Extremities of People with Stroke. Physical Therapy, 82, 25-34.

[25] Morris, S. (2002) Ashworth and Tardieu Scales: Their Clinical Relevance for Measuring Spasticity in Adult and Paediatric Neurological Populations. Physical Therapy Reviews, 7, 53-62. http://dx.doi.org/10.1179/108331902125001770

[26] Gracies, J.M., Burke, K., Clegg, N.J., Browne, R., Rushing, C., Fehlings, D., Matthews, D., Tilton, A. and Delgardo, M.R. (2010) Reliability of the Tardieu Scale for Assessing Spasticity in Children with Cerebral Palsy. Archives of Physical Medicine and Rehabilitation, 91,421-428. http://dx.doi.org/10.1016/j.apmr.2009.11.017

[27] Ben-Shabat, E., Palit, M., Fini, N.A., Brooks, C.T., Winter, A. and Holland, A.E. (2013) Intra- and Interrater Reliability of the Modified Tardieu Scale for the Assessment of Lower Limb Spasticity in Adults with Neurologic Injuries. Archives of Physical Medicine and Rehabilitation, 94, 2494-2501. http://dx.doi.org/10.1016/j.apmr.2013.06.026

[28] Haugh, A.B., Pandyan, A.D. and Johnson, G.R. (2006) A Systematic Review of the Tardieu Scale for the Measurement of Spasticity. Disability \& Rehabilitation, 28, 899-907. http://dx.doi.org/10.1080/09638280500404305

[29] Love, S.C., Gibson, N., Cole, J., Williams, N. and Blair, E. (2008) The Reliability of the Australian Spasticity Assessment Scale. Proceedings of the Australasian Academy of Cerebral Palsy and Developmental Medicine Conference, Brisbane, 30 May-2 June 2008, 28.

[30] Love, S., Gibson, N., Smith, N., Bear, N. and Blair, E. (2015) Reliability of the Australian Spasticity Assessment Scale (ASAS). Developmental Medicine \& Child Neurology, in Press.

[31] Altman, D.G. (1990) Practical Statistics for Medical Research. CRC Press, Boca Raton.

[32] Proske, U., Morgan, D.L. and Gregory, J.E. (1993) Thixotropy in Skeletal Muscle and in Muscle Spindles: A Review. Progress in Neurobiology, 41, 705-721. http://dx.doi.org/10.1016/0301-0082(93)90032-n

[33] Ansari, N.N., Naghdi, S., Hasson, S., Azarsa, M.H. and Azarnia, S. (2008) The Modified Tardieu Scale for the Measurement of Elbow Flexor Spasticity in Adult Patients with Hemiplegia. Brain Injury, 22, 1007-1012 http://dx.doi.org/10.1080/02699050802530557

[34] Kaya, T., Karatepe, A.G., Gunaydin, R., Koc, A. and Ercan, U.A. (2011) Inter-Rater Reliability of the Modified Ashworth Scale and Modified Modified Ashworth Scale in Assessing Poststroke Elbow Flexor Spasticity. International Journal of Rehabilitation Research, 34, 59-64. http://dx.doi.org/10.1097/MRR.0b013e32833d6cdf

[35] Li, F., Wu, Y. and Xiong, L. (2014) Reliability of a New Scale for Measurement of Spasticity in Stroke Patients. Journal of Rehabilitation Medicine, 46, 746-753. http://dx.doi.org/10.2340/16501977-1851 